\title{
Modulation of $\mathrm{CD}^{+}$and $\mathrm{CD}^{+}{ }^{+} \mathrm{T}$ Cell Function and Cytokine Responses in Strongyloides stercoralis Infection by Interleukin-27 (IL-27) and IL-37
}

\begin{abstract}
Rajamanickam Anuradha, a Saravanan Munisankar, ${ }^{a}$ Yukthi Bhootra, a Chandrakumar Dolla, ${ }^{b}$ Paul Kumaran, ${ }^{b}$ Thomas B. Nutman, ${ }^{c}$ Subash Babua,c

National Institutes of Health-NIRT-International Center for Excellence in Research, Chennai, Indiaa; National Institute for Research in Tuberculosis, Chennai, Indiab; Laboratory of Parasitic Diseases, NIAID, NIH, Bethesda, Maryland, USAc
\end{abstract}

ABSTRACT Strongyloides stercoralis infection is associated with diminished antigenspecific Th1- and Th17-associated responses and enhanced Th2-associated responses. Interleukin-27 (IL-27) and IL-37 are two known anti-inflammatory cytokines that are highly expressed in S. stercoralis infection. We therefore wanted to examine the role of IL-27 and IL-37 in regulating $\mathrm{CD}^{+}$and $\mathrm{CD}^{+}{ }^{+} \mathrm{T}$ cell responses in S. stercoralis infection. To this end, we examined the frequency of Th1/Tc1, Th2/Tc2, Th9/Tc9, Th17/ Tc17, and Th22/Tc22 cells in 15 S. stercoralis-infected individuals and 10 uninfected individuals stimulated with parasite antigen following IL-27 or IL-37 neutralization. We also examined the production of prototypical type 1, type 2, type 9, type 17, and type 22 cytokines in the whole-blood supernatants. Our data reveal that IL-27 or IL-37 neutralization resulted in significantly enhanced frequencies of Th1/Tc1, Th2/ Tc2, Th17/Tc17, Th9, and Th22 cells with parasite antigen stimulation. There was no induction of any $\mathrm{T}$ cell response in uninfected individuals following parasite antigen stimulation and IL-27 or IL-37 neutralization. Moreover, we also observed increased production of gamma interferon (IFN- $\gamma$ ), IL-5, IL-9, IL-17, and IL-22 and decreased production of IL-10 following IL-27 and IL-37 neutralization and parasite antigen stimulation in whole-blood cultures. Thus, we demonstrate that IL-27 and IL-37 limit the induction of particular $\mathrm{T}$ cell subsets along with cytokine responses in S. stercoralis infections, which suggest the importance of IL-27 and IL-37 in immune modulation in a chronic helminth infection.

KEYWORDS cytokines, helminths, T cells, immune mechanisms

nterleukin-27 (IL-27) and IL-37 belong to a family of anti-inflammatory cytokines known to downmodulate T cell and cytokine responses (1). IL-27 is a known regulator of Th1-, Th2-, and Th17-associated responses in various inflammatory settings. Indeed, IL-27 has been shown to antagonize the production of IL-1, inhibit Th2 and Th17 differentiation, and increase the production of IL-10 (2). IL-27 is also known to regulate Th1 and Th2 responses in murine Schistosoma mansoni (3) and Trichuris muris (4) infections. Similarly, IL-37 is also known to suppress inflammatory responses primarily by suppressing cytokine production through increased production of IL-10 (5). However, the role of IL-37 in helminth infections has not been explored.

Stongyloides stercoralis is a common helminth parasite infecting between 50 and 100 million people worldwide (6). Similar to other helminth infections, immune responses in $S$. stercoralis infection are characterized by relatively diminished antigen-specific Th1/Tc1 and Th17/Tc17 responses and relatively expanded Th2/Tc2 and Th9/Tc9 responses $(7,8)$. The modulation of Th1-, Th2-, and Th17-associated responses has been
Received 14 July 2017 Returned for modification 9 August 2017 Accepted 25 August 2017

Accepted manuscript posted online 5 September 2017

Citation Anuradha R, Munisankar S, Bhootra Y Dolla C, Kumaran P, Nutman TB, Babu S. 2017 Modulation of $\mathrm{CD}^{+}$and $\mathrm{CD} 8^{+} \mathrm{T}$ cell function and cytokine responses in Strongyloides stercoralis infection by interleukin-27 (IL-27) and IL-37. Infect Immun 85:e00500-17. https:// doi.org/10.1128/IAl.00500-17.

Editor Judith A. Appleton, Cornell University Copyright $\odot 2017$ American Society for Microbiology. All Rights Reserved. Address correspondence to Subash Babu sbabu@mail.nih.gov. 


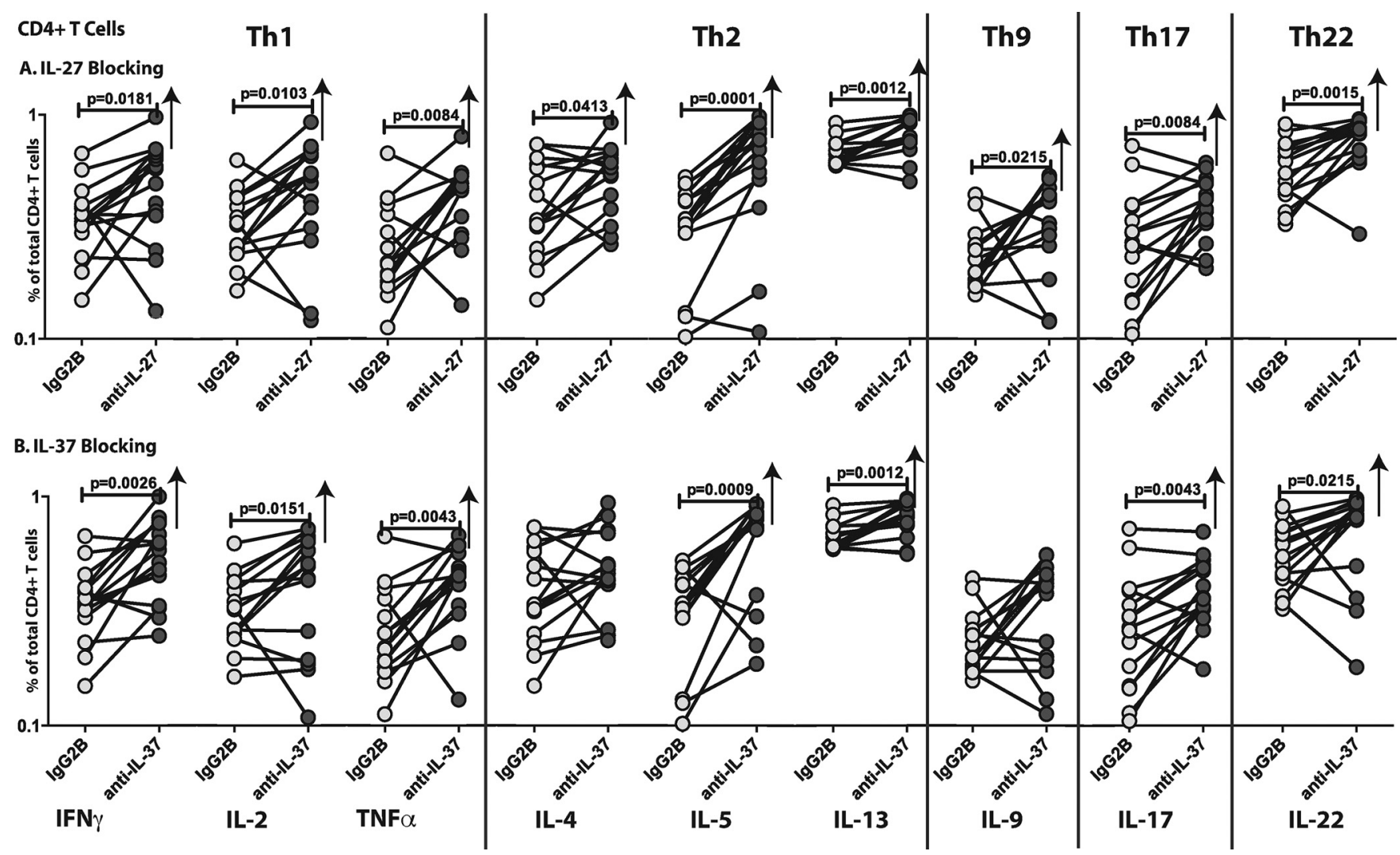

FIG 1 Altered frequencies of CD4+ Th1, Th2, Th9, Th17, and Th22 cells following neutralization of IL-27 and IL-37 in S. stercoralis infection. The NIE-stimulated frequencies of CD4+ Th1, Th2, Th9, Th17, and Th22 cells were measured by flow cytometry following neutralization of IL-27 (A), IL-37 (B), or isotype control antibody in S. stercoralis-infected individuals. The data are represented as line diagrams, with each line representing a single individual. $P$ values were calculated by the Wilcoxon signed-rank test followed by Holm's correction.

previously shown to be mediated by two regulatory cytokines: IL-10 and transforming growth factor $\beta$ (TGF- $\beta$ ) $(7,8)$. As we have shown previously, IL-27 and IL-37 are present at significantly higher levels in the circulation of $S$. stercoralis-infected individuals than in uninfected individuals, which revert back to normal levels following anthelmintic therapy (9). Thus, we sought to delineate the impact of these cytokines on T cell function in $S$. stercoralis infections. We demonstrate that IL-27 and IL-37 modulate Th1/Tc1, Th2/Tc2, and Th17/Tc17 responses primarily, with measureable effects on Th9 and Th22 responses as well.

\section{RESULTS}

Regulation of CD4 ${ }^{+} \mathbf{T}$ cell subsets by IL-27 and IL-37. To examine the effect of IL-27 and IL-37 on CD4 ${ }^{+}$T cells in S. stercoralis infections, we measured the frequencies of Th1 (gamma interferon [IFN- $\gamma$ ], tumor necrosis factor alpha [TNF- $\alpha$ ], or IL-2 expressing), Th2 (IL-4, IL-5, or IL-13 expressing), Th9 (IL-9 expressing), Th17 (IL-17 expressing), and Th22 (IL-22 expressing) cells following neutralization of IL-27 or IL-37 and stimulation with NIE antigen in S. stercoralis-infected $(n=15)$ and S. stercoralis-uninfected $(n=10)$ individuals. As shown in Fig. 1A, IL-27 neutralization resulted in significantly increased frequencies of CD4+ Th1, Th2, Th9, Th17, and Th22 cells. As shown in Fig. 1B, IL-37 neutralization resulted in significantly increased frequencies of Th1, Th2 (except IL-4), Th17, and Th22 cells. In addition, IL-27 or IL-37 neutralization had no significant effect on the $\mathrm{CD}^{+}{ }^{+} \mathrm{T}$ cell frequencies in response to NIE in S. stercoralis-uninfected individuals, and the frequencies were significantly lower than those in S. stercoralisinfected individuals (Table 1). Also, neutralization of IL-27 or IL-37 on unstimulated samples had no effect on $\mathrm{CD}^{+}{ }^{+} \mathrm{T}$ cell frequencies (data not shown). Representative whole-blood intracellular cytokine assay flow data from an infected individual showing 
TABLE 1 Frequencies of $\mathrm{CD}^{+}$and $\mathrm{CD}^{+}{ }^{+}$T cells based on cytokine responses in S. stercoralis-infected and -uninfected individuals ${ }^{a}$

\begin{tabular}{|c|c|c|c|c|c|c|}
\hline \multirow[b]{2}{*}{ Cytokine } & \multicolumn{2}{|c|}{$\begin{array}{l}\text { GM }(95 \% \mathrm{Cl}) \% \text { of expression by } \\
\text { CD4 }{ }^{+} \mathrm{T} \text { cells }\end{array}$} & \multirow[b]{2}{*}{$P$ value } & \multicolumn{2}{|c|}{$\begin{array}{l}\text { GM }(95 \% \mathrm{Cl}) \% \text { of expression by } \\
\text { CD8 }{ }^{+} \mathrm{T} \text { cells }\end{array}$} & \multirow[b]{2}{*}{$P$ value } \\
\hline & Infected & Uninfected & & Infected & Uninfected & \\
\hline \multicolumn{7}{|c|}{ IL-27 blocking } \\
\hline $\mathrm{IL}-2$ & $0.4322(0.6021)$ & $0.1436(0.1560)$ & 0.0001 & $0.2601(0.3029)$ & $0.1222(0.1281)$ & $<0.0001$ \\
\hline TNF- $\alpha$ & $0.4113(0.5198)$ & $0.1456(0.1609)$ & $<0.0001$ & $0.3597(0.5297)$ & $0.1623(0.2257)$ & 0.0043 \\
\hline IL-4 & $0.5144(0.6299)$ & $0.1436(0.1538)$ & $<0.0001$ & $0.4703(0.6425)$ & $0.1456(0.1578)$ & $<0.0001$ \\
\hline IL-9 & $0.3237(0.2456)$ & $0.1507(0.1665)$ & 0.0005 & $0.3952(0.5303)$ & $0.1414(0.1515)$ & $<0.0001$ \\
\hline IL-17 & $0.3874(0.4641)$ & $0.1279(0.1383)$ & $<0.0001$ & $0.3169(0.4695)$ & $0.1376(0.1550)$ & $<0.0001$ \\
\hline IL-22 & $0.7607(0.8985)$ & $0.1564(0.1666)$ & $<0.0001$ & $0.3371(0.3980)$ & $0.1365(0.1450)$ & $<0.0001$ \\
\hline \multicolumn{7}{|c|}{ IL-37 blocking } \\
\hline IFN- $\gamma$ & $0.5270(0.6631)$ & $0.1520(0.1692)$ & $<0.0001$ & $0.5325(0.6526)$ & $0.1502(0.1693)$ & $<0.0001$ \\
\hline IL-9 & $0.3057(0.4122)$ & $0.1507(0.1680)$ & 0.0004 & $0.4154(0.5372)$ & $0.1482(0.1620)$ & $<0.0001$ \\
\hline IL-17 & $0.3859(0.4678)$ & $0.1542(0.1730)$ & $<0.0001$ & $0.3234(0.4631)$ & $0.1420(0.1610)$ & 0.0001 \\
\hline IL-22 & $0.6752(0.8964)$ & $0.1386(0.1525)$ & $<0.0001$ & $0.3521(0.4094)$ & $0.1590(0.1745)$ & $<0.0001$ \\
\hline
\end{tabular}

${ }^{a}$ Cytokine responses are shown as geometric means (GM) with $95 \%$ confidence intervals (Cl) in parentheses.

expression of $\mathrm{CD}^{+} \mathrm{T}$ cell cytokines are shown in Fig. $\mathrm{S} 1$ in the supplemental material. Thus, IL-27 and/or IL-37 appear to act on CD4 ${ }^{+} \mathrm{T}$ cells by downregulating antigenspecific Th1, Th2, Th9, Th17, and Th22 responses in S. stercoralis infection.

Regulation of $\mathrm{CD8}^{+} \mathbf{T}$ cell subsets by IL-27 and IL-37. To examine the effect of IL-27 and IL-37 on CD8 ${ }^{+}$T cells in S. stercoralis infections, we measured the frequencies of Tc1 (IFN- $\gamma$, TNF- $\alpha$, or IL-2 expressing), Tc2 (IL-4, IL-5, or IL-13 expressing), TC9 (IL-9 expressing), Tc17 (IL-17 expressing), and Tc22 (IL-22 expressing) CD8 ${ }^{+}$T cells following neutralization of IL-27 or IL-37 and stimulation with NIE in S. stercoralis-infected $(n=15)$ individuals. As shown in Fig. 2A, IL-27 neutralization resulted in significantly increased frequencies of Tc1 (except TNF- $\alpha$ ), Tc2, Tc9, Tc17, and Tc22 cells. As shown in Fig. 2B, IL-37 neutralization resulted in significantly increased frequencies of Tc1, Tc2 (except IL-4), Tc9, Tc17, and Tc22 cells. In addition, IL-27 or IL-37 neutralization had no significant effect on the $\mathrm{CD}^{+}{ }^{+} \mathrm{T}$ cell frequencies in response to NIE in S. stercoralisuninfected individuals, and the frequencies were significantly lower than those in $S$. stercoralis-infected individuals (Table 1). Also, neutralization of IL-27 or IL-37 on unstimulated samples had no effect on $\mathrm{CD}^{+} \mathrm{T}$ cell frequencies (data not shown). Representative whole-blood intracellular cytokine assay flow data from an infected individual showing expression of $\mathrm{CD}^{+} \mathrm{T}$ cell cytokines are shown in Fig. S1. Thus, IL-27 and IL-37 both appear to act on CD8 ${ }^{+}$T cells by downregulating Tc1, Tc2, Tc9, Tc17, and Tc22 responses in $S$. stercoralis infection.

Regulation of cytokine responses by IL-27 and IL-37. To examine the effect of IL-27 and IL-37 on total cytokine responses in S. stercoralis infections, we measured the levels of type 1 (IFN- $\gamma$ ), type 2 (IL-5), type 9 (IL-9), Tr1 (IL-10), type 17 (IL-17), and type 22 (IL-22) cytokines following neutralization of IL-27 or IL-37 and stimulation with NIE in S. stercoralis-infected individuals. As shown in Fig. 3A, IL-27 neutralization resulted in significantly increased levels of IFN- $\gamma$ (geometric mean [GM] of $506.8 \mathrm{pg} / \mathrm{ml}$ in the isotype control versus $584.7 \mathrm{pg} / \mathrm{ml}$ in anti-IL-27), IL-5 (GM of $209.1 \mathrm{pg} / \mathrm{ml}$ versus 241.4 $\mathrm{pg} / \mathrm{ml}$ ), IL-9 (GM of $272.5 \mathrm{pg} / \mathrm{ml}$ versus $309.9 \mathrm{pg} / \mathrm{ml}$ ), IL-17 (GM of $198.1 \mathrm{pg} / \mathrm{ml}$ versus $255.5 \mathrm{pg} / \mathrm{ml}$ ), and IL-22 (GM of $251.2 \mathrm{pg} / \mathrm{ml}$ versus $279 \mathrm{pg} / \mathrm{ml}$ ) and significantly decreased levels of IL-10 (GM of $149.8 \mathrm{pg} / \mathrm{ml}$ versus $126.4 \mathrm{pg} / \mathrm{ml}$ ). Similarly, as shown in Fig. 3B, IL-37 neutralization resulted in significantly increased levels of IFN- $\gamma$ (GM of $506.8 \mathrm{pg} / \mathrm{ml}$ versus $560.6 \mathrm{pg} / \mathrm{ml}$ ), IL-5 (GM of $209.1 \mathrm{pg} / \mathrm{ml}$ versus $238.2 \mathrm{pg} / \mathrm{ml}$ ), IL-17 (GM 


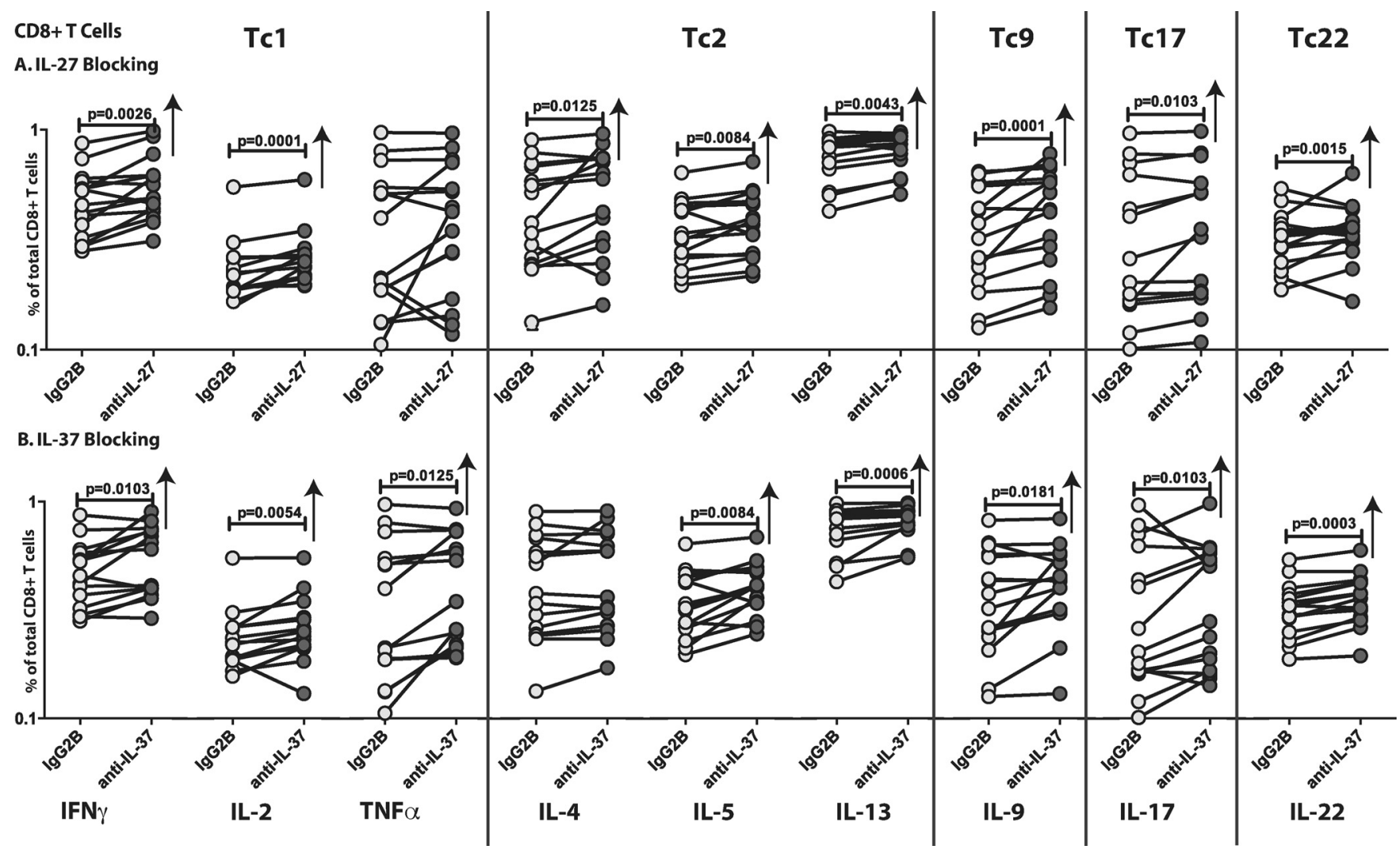

FIG 2 Altered frequencies of CD8 ${ }^{+}$Tc1, Tc2, TC9, Tc17, and TC22 cells following neutralization of IL-27 and IL-37 in S. stercoralis infection. The NIE-stimulated frequencies of $\mathrm{CD}^{+} \mathrm{Tc1}, \mathrm{Tc17}, \mathrm{Tc22}, \mathrm{Tc2}$, Tc9, and Tr1 cells were measured by flow cytometry following neutralization of IL-27 (A), IL-37 (B), or isotype control antibody in $S$. stercoralis-infected individuals. The data are represented as line diagrams, with each line representing a single individual. $P$ values were calculated by the Wilcoxon signed-rank test followed by Holm's correction.

of $198.1 \mathrm{pg} / \mathrm{ml}$ versus $256.9 \mathrm{pg} / \mathrm{ml}$ ), and IL-22 (GM of $251.2 \mathrm{pg} / \mathrm{ml}$ versus $285.1 \mathrm{pg} / \mathrm{ml}$ ) and significantly decreased levels of IL-10 (GM of $149.8 \mathrm{pg} / \mathrm{ml}$ versus $120.2 \mathrm{pg} / \mathrm{ml}$ ). In addition, IL-27 or IL-37 neutralization had no significant effect on the cytokine responses to NIE in S. stercoralis-uninfected individuals. Thus, IL-27 and/or IL-37 appears to regulate many cytokine responses in S. stercoralis infection.

\section{DISCUSSION}

Negative regulation of the immune response involving suppressor cytokines is an intrinsic part of the immune system, and regulatory cytokines typically provide a feedback mechanism to prevent excessive immunity and collateral host damage (1). Two of the more recently described cytokines in this group are IL-27 and IL-37 (1). IL-27 belongs to the IL-12 family of cytokines but predominantly exhibits anti-inflammatory properties (10). Although initially described as a Th1-promoting factor (11), it has been shown more recently to downmodulate immune responses in a variety of settings (12). Thus, mice deficient in the receptor for IL-27 (IL-27R) are known develop immunemediated pathology and lethal inflammation following infection with the parasites, Toxoplasma gondii and Trypanosoma cruzi $(13,14)$. In contrast, in the setting of helminth infection, IL-27 has been shown to be an important negative regulator of Th2 responses in that IL-27R-deficient mice show enhanced resistance to intestinal helminth infections $(4,15)$. IL-37 belongs to the IL-1 family of cytokines, a family that also includes the proinflammatory cytokines IL- $1 \alpha$, IL- $\beta$, and IL-18 (16). Unlike the other IL-1 family members, IL-37 clearly functions as an anti-inflammatory cytokine, as seen in studies of mice transgenically expressing human IL-37 that are protected from lipopolysaccharide (LPS)-induced septic shock and dextran sulfate-induced colitis $(16,17)$. Moreover, IL-37 has also been reported to suppress antigen-specific $T$ cell response immunity in mice 


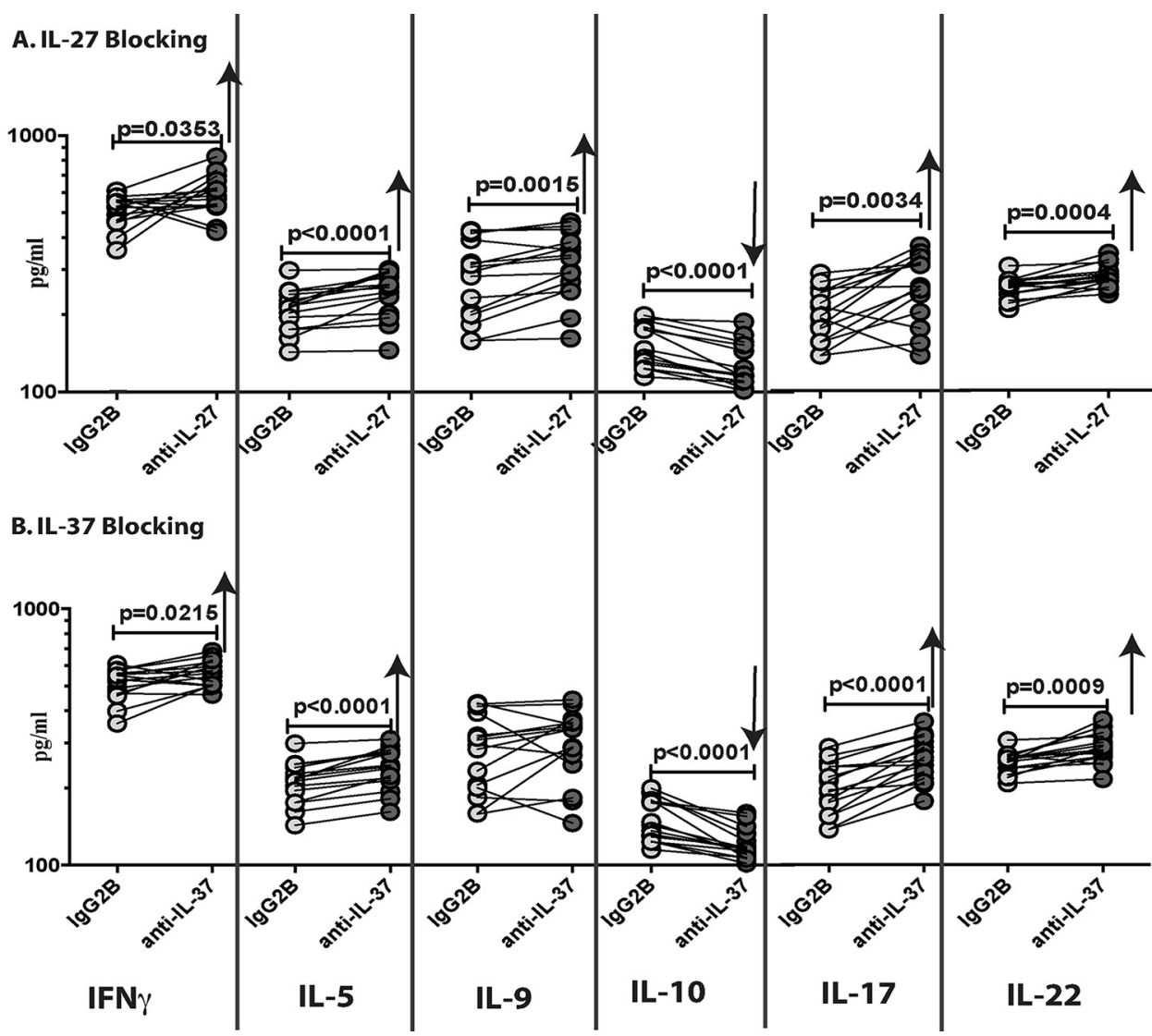

FIG 3 Altered levels of different cytokines following neutralization of IL-27 and IL-37 in S. stercoralis infection. The NIE-stimulated levels of IFN- $\gamma$, IL-5, IL-9, IL-10, IL-17, and IL-22 were measured by ELISA in whole-blood supernatants following neutralization of IL-27 (A), IL-37 (B), or isotype control antibody in S. stercoralis-infected individuals ( $n=$ 15). The data are represented as line diagrams, with each line representing a single individual. $P$ values were calculated by the Wilcoxon signed-rank test followed by Holm's correction.

through the induction of tolerogenic dendritic cells (18). In addition, IL-37 has been shown to inhibit the production of proinflammatory cytokines in systemic lupus erythematosus (19).

Having previously shown that IL-27 and IL-37 are two among a large panel of anti-inflammatory cytokines induced systemically in S. stercoralis infection and whose induction is reversed following definitive anthelmintic therapy (9), we sought to understand the role of IL-27 and IL-37 in S. stercoralis infection. Our findings reveal the role played by IL-27 in S. stercoralis infection by demonstrating its effect on all the subsets of both $\mathrm{CD}^{+}$and $\mathrm{CD}^{+}{ }^{+} \mathrm{T}$ cells responding to parasite antigen. Thus, IL-27 clearly downmodulates the Th1/Tc1, Th2/Tc2, Th9/Tc9, Th17/Tc17, and Th22/Tc22 arms of $\mathrm{T}$ cell subsets responding to parasite antigen. In addition, our data reveal a novel role for IL-37 in human helminth infections. However, to a lesser extent, IL-37 also induces downmodulation of the frequencies of Th1/Tc1, Th2/Tc2, Tc9, Th17/Tc17, and Th22/ Tc22. Interestingly, this effect on the downregulation of $\mathrm{CD}^{+}$and $\mathrm{CD}^{+} \mathrm{T}$ cell cytokine responses is specific to $S$. stercoralis infection, since neutralization of IL-27 or IL-37 had no significant effect on $T$ cell cytokine responses of uninfected individuals. In addition, T cells from S. stercoralis-infected individuals in the absence of parasite antigen stimulation do not exhibit any modulation of cytokine responses, as would be expected from the absence of a stimulus. However, it is of interest that while Th1, Th17, and Th22 responses are typically downmodulated in helminth infections, including S. stercoralis infection, Th2 and Th9 responses are commonly upregulated. Therefore, our data would imply that although IL-27 and IL-37 do function by downregulating Th2 and Th9 
responses, they fail to effectively do so in the context of S. stercoralis infections and perhaps other helminth infections as well.

We also demonstrate a plausible pathway by which IL-27 and IL-37 can modulate cytokine responses in S. stercoralis infection. Our analysis of IL-27 and IL-37 neutralization on the total levels of cytokines produced by whole-blood supernatants of $S$. stercoralis-infected individuals clearly reveals a marked downregulation of IL-10 production. While IL-10 is known to function downstream of IL-27 and mediate its regulatory function (20), a role for IL-10 in IL-37-mediated cytokine regulation has not been explored. Our data suggest that the upregulation of IL-10 by IL-27 and IL-37 could potentially account for a negative-feedback loop to induce downmodulation of $\mathrm{CD}^{+}$ and $\mathrm{CD}^{+} \mathrm{T}$ cell responses in this chronic helminth infection.

While IL-27 is a known modulator of Th1, Th2, Th9, and Th17 responses in different settings (10), this is the first study (to our knowledge) to demonstrate an effect on all of these responses in a single infection. In addition, we also elucidate a new role for IL-27 in the modulation of Th22 responses. IL-27 is known to induce IL-10 production and also to antagonize the expression of T-bet, Gata-3, and RORC, the master transcription factors for Th1, Th2, and Th17 responses (10), likely providing a mechanism of the downmodulation of these responses in our study as well. In contrast, the role of IL-37 in T cell-mediated responses has not been examined in detail. Increased levels of IL-37 have been reported in several human disease conditions, including rheumatoid arthritis and inflammatory bowel disease, but IL-37 has been mostly shown to downmodulate proinflammatory cytokines from antigen-presenting cells in these diseases (5). Our study is thus one of the first studies to examine the role of IL-37 in the regulation of $\mathrm{CD}^{+}$and $\mathrm{CD}^{+}{ }^{+} \mathrm{T}$ cell responses in a parasitic infection, and we demonstrate an important effect in downmodulating the various arms of $\mathrm{T}$ cell differentiation and/or activation in S. stercoralis infection. Whether IL-37 also modulates the expression of master transcription factors in $\mathrm{T}$ cell responses remains to be elucidated. It is also possible that IL-27 and IL-37 act on TGF- $\beta$ as a downstream regulatory effector to mediate these effects. Our study did not examine the cellular sources of IL-27 and IL-37 and also did not examine whether the effect of IL-27 and IL-37 is directly on T cells or mediated via antigen-presenting cells. Another limitation is that the frequency of $T$ cells expressing the various cytokines in the flow cytometry assays is small, although this is corroborated by the enzyme-linked immunosorbent assay (ELISA) data.

Our data provide evidence of an important role for IL-27 and IL-37 as regulatory cytokines modulating the antigen-specific $T$ cell response in a helminth infection. This suggests that IL-27 and IL-37 can be added to a growing list of cytokine effectors that play a regulatory role in helminth infections.

\section{MATERIALS AND METHODS}

Study population. We studied a group of 15 clinically asymptomatic, S. stercoralis-infected individuals and 10 uninfected control individuals in Tamil Nadu, south India. All infected individuals were NIE ELISA positive and because they had not received any anthelmintic treatment prior to enrollment in this study were deemed infected with S. stercoralis. Uninfected control individuals were negative by the NIE ELISA. All individuals were examined as part of a natural history study with the protocol approved by the Institutional Review Boards of both the National Institutes of Allergy and Infectious Diseases and the National Institute for Research in Tuberculosis (NCT00001230), and informed written consent was obtained from all participants.

Parasite antigen. Recombinant NIE antigen, a previously characterized immunodominant, 31-kDa S. stercoralis larval antigen, was used as the parasite antigen (21).

In vitro culture. Whole blood from S. stercoralis-infected and -uninfected individuals was cultured in the presence of anti-IL-27, anti-IL-37, or isotype control antibody (all at $5 \mu \mathrm{g} / \mathrm{ml}$ ) (R\&D Systems) with NIE antigen $(10 \mu \mathrm{g} / \mathrm{ml})$ for $18 \mathrm{~h}$. We also performed IL-27 and IL-37 neutralization on unstimulated whole blood from S. stercoralis-infected individuals as well. Briefly, whole blood was diluted 1:1 with RPMI 1640 medium, supplemented with penicillin-streptomycin $(100 \mathrm{U} / 100 \mathrm{mg} / \mathrm{ml})$, L-glutamine $(2 \mathrm{mM})$, and HEPES (10 mM) (all from Invitrogen, San Diego, CA), and placed in 12-well tissue culture plates (Costar, Corning, Inc., NY). Fastlmmune brefeldin A solution (10 $\mu \mathrm{g} / \mathrm{ml}$ ) (BD Biosciences) was added $2 \mathrm{~h}$ before the end of the culture. Whole-blood supernatants were collected before the addition of brefeldin A. Whole blood was then centrifuged and washed with cold phosphate-buffered saline (PBS), and then $1 \times$ fluorescenceactivated cell sorter (FACS) lysing solution (BD Biosciences, San Diego, CA) was added. The cells were 
fixed using cytofix/cytoperm buffer (BD Biosciences, San Diego, CA), cryopreserved, and stored at $-80^{\circ} \mathrm{C}$ until use.

Intracellular cytokine staining. The cells were thawed and washed with PBS first and PBS-1\% bovine serum albumin (BSA) next and then stained with antibodies expressed on the cell surface for 30 to $60 \mathrm{~min}$. The following antibodies were used for surface marker staining: CD3-Amcyan; CD4allophycocyanin (APC)-conjugated H7, and CD8-phycoerythrin (PE)-conjugated Cy7 (all from BD Biosciences). The cells were washed, permeabilized with BD Perm/Wash buffer (BD Biosciences), and stained with intracellular cytokines for an additional $30 \mathrm{~min}$ before washing and acquisition. The following cytokine antibodies were used: CD3-AmCyan, clone SK7 (BD Biosciences); CD4-PE-Cy7, clone SK3 (BD Pharmingen); CD8-APC-H7, clone SK1 (BD Pharmingen); IFN- $\gamma-\mathrm{PE}$, clone 4S.B3 (BD Pharmingen); TNF$\alpha$-fluorescein isothiocyanate (FITC), clone 6401.1111 (BD Biosciences); IL-2-APC, clone MQ1-17H12 (eBioscience); IL-4-FITC, clone MP4-25D2 (RUO) (BD Biosciences); IL-5-APC, TRFK5 (RUO) (BD Biosciences); IL-9-PE, clone MH9A3 (BD Pharmingen); IL-17-FITC, clone CZ8-23G1 (Miltenyi Biotech); and IL-22-PE, clone 142928 (R\&D Systems). Flow cytometry was performed on a FACSCanto II flow cytometer with FACSDiva software v.6 (Becton Dickinson). The lymphocyte gating was set by forward and side scatter, and 100,000 gated lymphocyte events were acquired. Data were collected and analyzed using Flow Jo software (TreeStar). All data are depicted as frequencies denoted by the percentage of $\mathrm{CD}^{+}{ }^{+}$and CD8 ${ }^{+} \mathrm{T}$ cells expressing the respective cytokine(s).

ELISA. Plasma and culture supernatant levels of IFN- $\gamma$, IL-5, IL-10, IL-17, IL-22 (all R\&D Systems), and IL-9 (eBioscience) were measured by ELISA, according to the manufacturer's instructions. All samples were run in duplicates.

Statistical analysis. Data analyses were performed using GraphPad PRISM (GraphPad Software, Inc., San Diego, CA). Geometric means (GM) were used for measurements of central tendency. Statistically significant differences were analyzed using the Wilcoxon signed-rank test followed by Holm's correction for multiple comparisons.

\section{SUPPLEMENTAL MATERIAL}

Supplemental material for this article may be found at https://doi.org/10.1128/IAI .00500-17.

SUPPLEMENTAL FILE 1, PDF file, 1.7 MB.

\section{ACKNOWLEDGMENTS}

We thank the staff of Department of Epidemiology, NIRT, for valuable assistance in recruiting the patients for this study and M. Satiswaran, J. Jeevan, Prabbu Balakrishnan, Pavan Kumar, and Jovvian George of the NIH-ICER for technical assistance.

This work was supported by the Intramural Research Program of the Division of Intramural Research, National Institute of Allergy and Infectious Diseases, National Institutes of Health. The funders had no role in study design, data collection and interpretation, or the decision to submit the work for publication.

The authors report no competing interests, and none of the authors has a financial relationship with a commercial entity that has an interest in the subject of the study.

\section{REFERENCES}

1. Banchereau J, Pascual V, O'Garra A. 2012. From IL-2 to IL-37: the expanding spectrum of anti-inflammatory cytokines. Nat Immunol 13:925-931. https://doi.org/10.1038/ni.2406.

2. Hunter CA, Kastelein R. 2012. Interleukin-27: balancing protective and pathological immunity. Immunity 37:960-969. https://doi.org/10.1016/j .immuni.2012.11.003.

3. Shainheit MG, Saraceno R, Bazzone LE, Rutitzky LI, Stadecker MJ. 2007. Disruption of interleukin-27 signaling results in impaired gamma interferon production but does not significantly affect immunopathology in murine schistosome infection. Infect Immun 75:3169-3177. https://doi .org/10.1128/IAI.01053-06.

4. Artis D, Villarino A, Silverman $M$, He W, Thornton EM, Mu S, Summer $S$, Covey TM, Huang E, Yoshida H, Koretzky G, Goldschmidt M, Wu GD, de Sauvage F, Miller HR, Saris CJ, Scott P, Hunter CA. 2004. The IL-27 receptor (WSX-1) is an inhibitor of innate and adaptive elements of type 2 immunity. J Immunol 173:5626-5634. https://doi.org/10.4049/ jimmunol.173.9.5626.

5. Dinarello CA, Nold-Petry C, Nold M, Fujita M, Li S, Kim S, Bufler P. 2016. Suppression of innate inflammation and immunity by interleukin-37. Eur J Immunol 46:1067-1081. https://doi.org/10.1002/eji.201545828.

6. Nutman TB. 2016. Human infection with Strongyloides stercoralis and other related Strongyloides species. Parasitology 144:263-273. https:// doi.org/10.1017/S0031182016000834.
7. Anuradha R, Munisankar S, Bhootra Y, Jagannathan J, Dolla C, Kumaran P, Nutman TB, Babu S. 2016. IL-10- and TGFbeta-mediated Th9 responses in a human helminth infection. PLoS Negl Trop Dis 10:e0004317. https:// doi.org/10.1371/journal.pntd.0004317.

8. Anuradha R, Munisankar S, Dolla C, Kumaran P, Nutman TB, Babu S. 2015 Parasite antigen-specific regulation of Th1, Th2, and Th17 responses in Strongyloides stercoralis infection. J Immunol 195:2241-2250. https://doi .org/10.4049/jimmunol.1500745.

9. Anuradha R, Munisankar S, Bhootra Y, Jagannathan J, Dolla C, Kumaran P, Shen K, Nutman TB, Babu S. 2015. Systemic cytokine profiles in Strongyloides stercoralis infection and alterations following treatment. Infect Immun 84:425-431. https://doi.org/10.1128/IAI.01354-15.

10. Yoshida $\mathrm{H}$, Hunter CA. 2015. The immunobiology of interleukin-27. Annu Rev Immunol 33:417-443. https://doi.org/10.1146/annurev-immunol -032414-112134.

11. Brombacher F, Kastelein RA, Alber G. 2003. Novel IL-12 family members shed light on the orchestration of Th1 responses. Trends Immunol 24:207-212. https://doi.org/10.1016/S1471-4906(03)00067-X.

12. Stumhofer JS, Hunter CA. 2008. Advances in understanding the antiinflammatory properties of IL-27. Immunol Lett 117:123-130. https://doi .org/10.1016/j.imlet.2008.01.011.

13. Hamano S, Himeno K, Miyazaki Y, Ishii K, Yamanaka A, Takeda A, Zhang M, Hisaeda H, Mak TW, Yoshimura A, Yoshida H. 2003. WSX-1 is required 
for resistance to Trypanosoma cruzi infection by regulation of proinflammatory cytokine production. Immunity 19:657-667. https://doi.org/ 10.1016/S1074-7613(03)00298-X.

14. Villarino A, Hibbert L, Lieberman L, Wilson E, Mak T, Yoshida H, Kastelein RA, Saris C, Hunter CA. 2003. The IL-27R (WSX-1) is required to suppress T cell hyperactivity during infection. Immunity 19:645-655. https://doi .org/10.1016/S1074-7613(03)00300-5.

15. Bancroft AJ, Humphreys NE, Worthington JJ, Yoshida H, Grencis RK. 2004. WSX-1: a key role in induction of chronic intestinal nematode infection. J Immunol 172:7635-7641. https://doi.org/10.4049/jimmunol.172.12.7635.

16. Nold MF, Nold-Petry CA, Zepp JA, Palmer BE, Bufler P, Dinarello CA. 2010. IL-37 is a fundamental inhibitor of innate immunity. Nat Immunol 11: 1014-1022. https://doi.org/10.1038/ni.1944.

17. McNamee EN, Masterson JC, Jedlicka P, McManus M, Grenz A, Collins CB, Nold MF, Nold-Petry C, Bufler P, Dinarello CA, Rivera-Nieves J. 2011. Interleukin 37 expression protects mice from colitis. Proc Natl Acad Sci U S A 108:16711-16716. https://doi.org/10.1073/pnas.1111982108.
18. Luo $Y$, Cai X, Liu S, Wang S, Nold-Petry CA, Nold MF, Bufler P, Norris D, Dinarello CA, Fujita M. 2014. Suppression of antigen-specific adaptive immunity by IL-37 via induction of tolerogenic dendritic cells. Proc Natl Acad Sci U S A 111:15178-15183. https://doi.org/10.1073/ pnas. 1416714111.

19. Ye L, Ji L, Wen Z, Zhou Y, Hu D, Li Y, Yu T, Chen B, Zhang J, Ding L, Du J, Huang Z. 2014. IL-37 inhibits the production of inflammatory cytokines in peripheral blood mononuclear cells of patients with systemic lupus erythematosus: its correlation with disease activity. J Transl Med 12:69. https://doi.org/10.1186/1479-5876-12-69.

20. Pot C, Apetoh L, Awasthi A, Kuchroo VK. 2011. Induction of regulatory $\mathrm{Tr} 1$ cells and inhibition of $\mathrm{T}(\mathrm{H}) 17$ cells by IL-27. Semin Immunol 23 : 438-445. https://doi.org/10.1016/j.smim.2011.08.003.

21. Abraham D, Hess JA, Mejia R, Nolan TJ, Lok JB, Lustigman S, Nutman TB. 2011. Immunization with the recombinant antigen Ss-IR induces protective immunity to infection with Strongyloides stercoralis in mice. Vaccine 29:8134-8140. https://doi.org/10.1016/j.vaccine.2011.08.030. 